\title{
FURTHER DATES OF HEAVY METAL CONTENT ON THE SOIL AND VEGETATION OF AGGTELEK KARST (HUNGARY)
}

\author{
NOVE MERITVE VSEBNOSTI TEŽKIH KOVIN V PRSTI IN \\ VEGETACIJI AGTELEŠKEGA KRASA (MADŽARSKA)
} RITA KASZALA ${ }^{1} \&$ ILONA BÁRÁNY-KEVEI ${ }^{1}$ \& KLÁRA POLYÁK²

${ }^{1}$ University of Szeged, Department of Climatology and Landscape Ecology, 6722. Szeged, Egyetem u. 2.

${ }^{2}$ University of Veszprém, Department of Earth- and Environmental Science, 8200.Veszprém, Egyetem u. 10. e-mail: rita@geo.u-szeged.hu 


\section{Abstract}

UDC: 504.05:546.3/.9(439)

Rita Kaszala \& Ilona Bárány-Kevei \& Klára Polyák: Further dates of heavy metal content on the soil and vegetation of Aggtelek Karst (Hungary)

During the previous investigation of the Aggtelek Karst we published physical and chemical parameters of the soils and the microelement content of the greenery. The presentation now gives further information about the soil (e.g. EDTA soluble heavy metal content), and the greenery (more species, more elements). The presentation shows the relationship between the heavy metal content of the karstic soil, and the sprout of plants.

Key words: karstic soil, heavy metals, Aggtelek karst, Hungary

Izvleček

UDK: 504.05:546.3/.9(439)

Rita Kaszala \& Ilona Bárány-Kevei \& Klára Polyák: Nove meritve vsebnosti težkih kovin v prsti in vegetaciji Agteleškega krasa (Madžarska).

O fizikalnih in kemičnih lastnostih prsti ter vsebnosti mikroelementov v rastlinju na območju Agteleškega krasa smo že poročali. V članku predstavimo nove meritve vsebnosti - v EDTA topnih - težkih kovin v prsti in meritve dodatnih elementov $\mathrm{v}$ različnih rastlinskih vrstah. Prikažemo tudi odnos med vsebnostjo težkih kovin v kraški prsti ter rastlinah in njihovimi poganjki.

Ključne besede: kraška prst, težke kovine, Agteleški kras, Madžarska. 


\section{INTRODUCTION}

During our investigation we determine the metal content of the soils and the greenery in the catchments basin of the Béke cave in Aggtelek Karstic area. This examination is a join research which is going on in the karst area in Aggtelek in order to survey the state of the karstic soils (mechanical compound, chemical and physical characteristics, content of the uptake able heavy metal). For examine the behaviour of the heavy metals in the soil one the qualities which are affected by the puffer capacity of the soils. These are the $\mathrm{pH}$ value, content of clay mineral- and organic matter, and the quality of the clay minerals, which are very important components also in the aspect of the heavy metal mobility in the soil. If the soil has sufficient $\mathrm{pH}$ value and the organic matter- and clay mineral contents are high enough, it can adsorb high quantity ions hereby decreasing the mobility of the ions. The heavy metal ions which get into the soil solution are available for the greenery, and through the shallow soil layer can get to the karstic water system which has a very important role in the drinking water system.

\section{DATAS OF THE INVESTIGATED AREA}

Our investigation takes place in the catchments basin of Béke cave at Aggtelek Karsts in Hungary. The examined area is about $10 \mathrm{~km}^{2}$, which has very variable geology. We can divide into the area two parts: the northern part is a non-covered karstic area, in contrast with the southern part which is a covered with pennon sediments. We can observe this duality in the developed soil at parent material. In the northern part of the area you can find mainly reddish clay remained soils which are rich in clay minerals and brown forest soils. On the other hand in the covered karst area we can find bright, yellowish-brown coloured soils, which contain loam and sand (remain like terra fusca).

From the point of view of phytogeography the catchments basin belongs to Pannonic Floraprovincial and the Floradistrict of Tornense. The vegetation of the area is very diverse. We can find a steppe-grassland (Salvio festucetum rupicole) a typical karst-shrub forest and extrazonal beech wood here. We can meet mainly natural forests here, but there are traces of the human effects on it. On the southern part of the area we can see impenetrable scrub built up from juniper and blackthorn. On that place there were forests, but after the deforestation the reforestation was not ensued because of the microclimate. The human effects become visible of the planted pine forests also. The warm preferable cornel-oak forest (Corno Quercetum Pubescenti Petrea) spread on a big part of the area. For our investigation we chose plant species which are widespread on the area an in this way we can get comparable information about the whole area. Oak, hornbeam, cornel leaves and grass were collected.

\section{METHODS}

We were collecting both soil and greenery samples from each sample point during the summer of 2003. The soils samples came from two depths: one from the surface $(0-10 \mathrm{~cm})$ and the other came from $20-30 \mathrm{~cm}$ depths. We determined $\mathrm{pH}$, organic matter-content, acid- and EDTA soluble heavy metal content from the soils.

The acid soluble heavy metal-content was determined after digestion with acid mixture $\left(\mathrm{HNO}_{3}\right.$ $\mathrm{H}_{2} \mathrm{O}_{2}-\mathrm{HClO}_{4}$ ) and the greenery metal content after digestion with $\mathrm{HNO}_{3}$ and $\mathrm{HClO}_{4}$. (ROWELL, D.L. 1994). 
The EDTA soluble metal content was determined after shaking with 0.02M EDTA solution $(\mathrm{pH}=4.65 \pm 0.05)$ and filtered it. (LAKANEN, E.- ERVIÖ, R. 1971) The manganese, iron, magnesium, calcium, potassium, aluminium content of the solutions which made from the greenery and the soils were determined by ICP-AES techniques, and the copper and zinc by FAAS techniques. The measurements took place in the University of Veszprém.

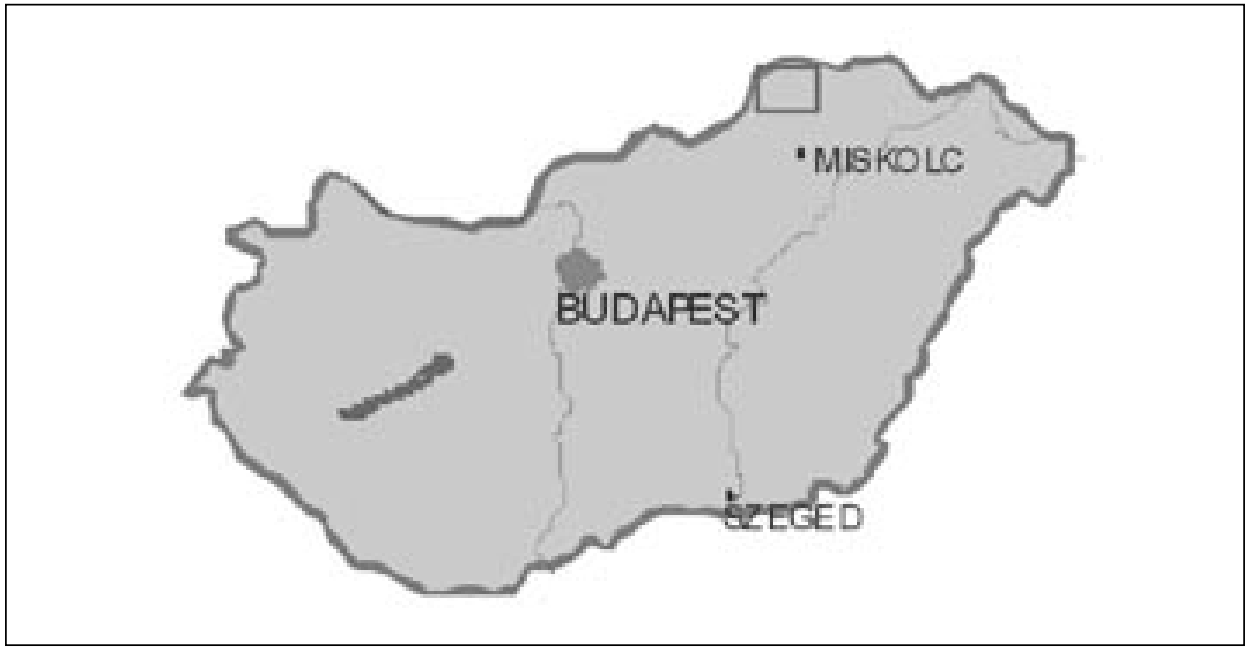

Map I. Map of Hungary.

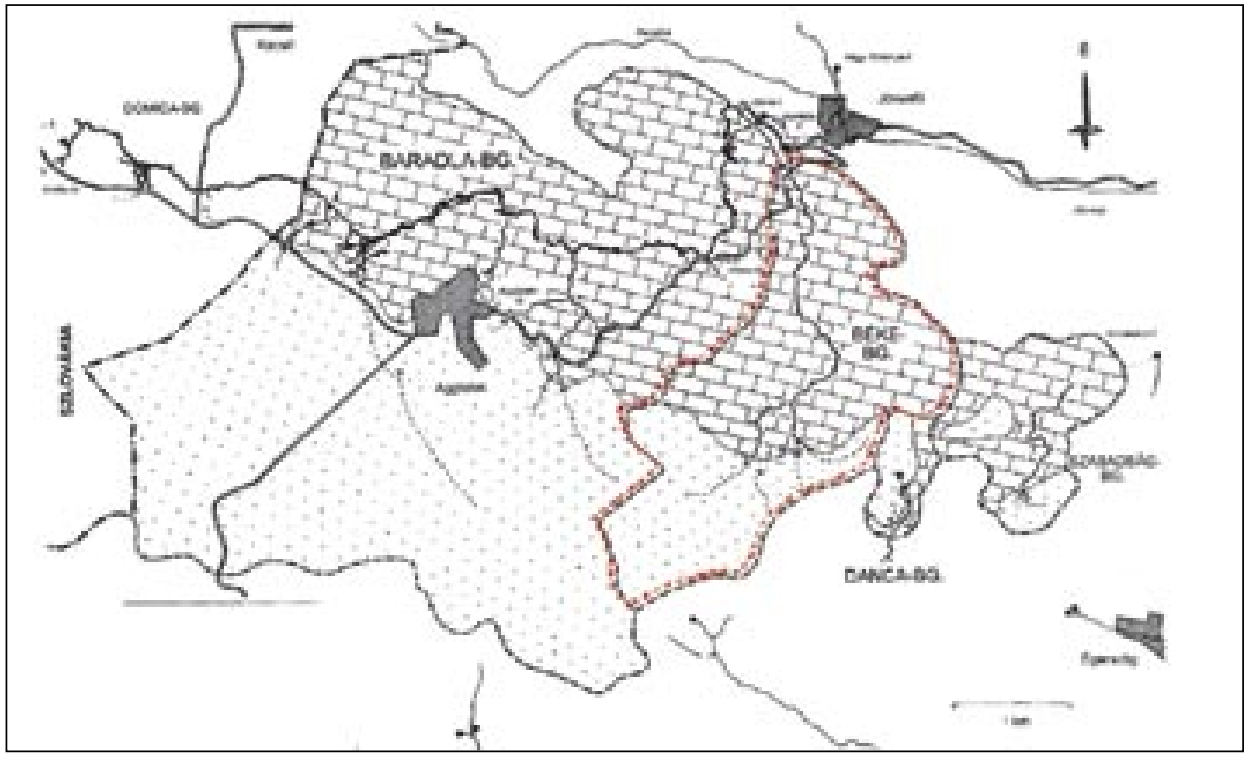

Map II.. The geological Map of Aggtelek Mountain. 


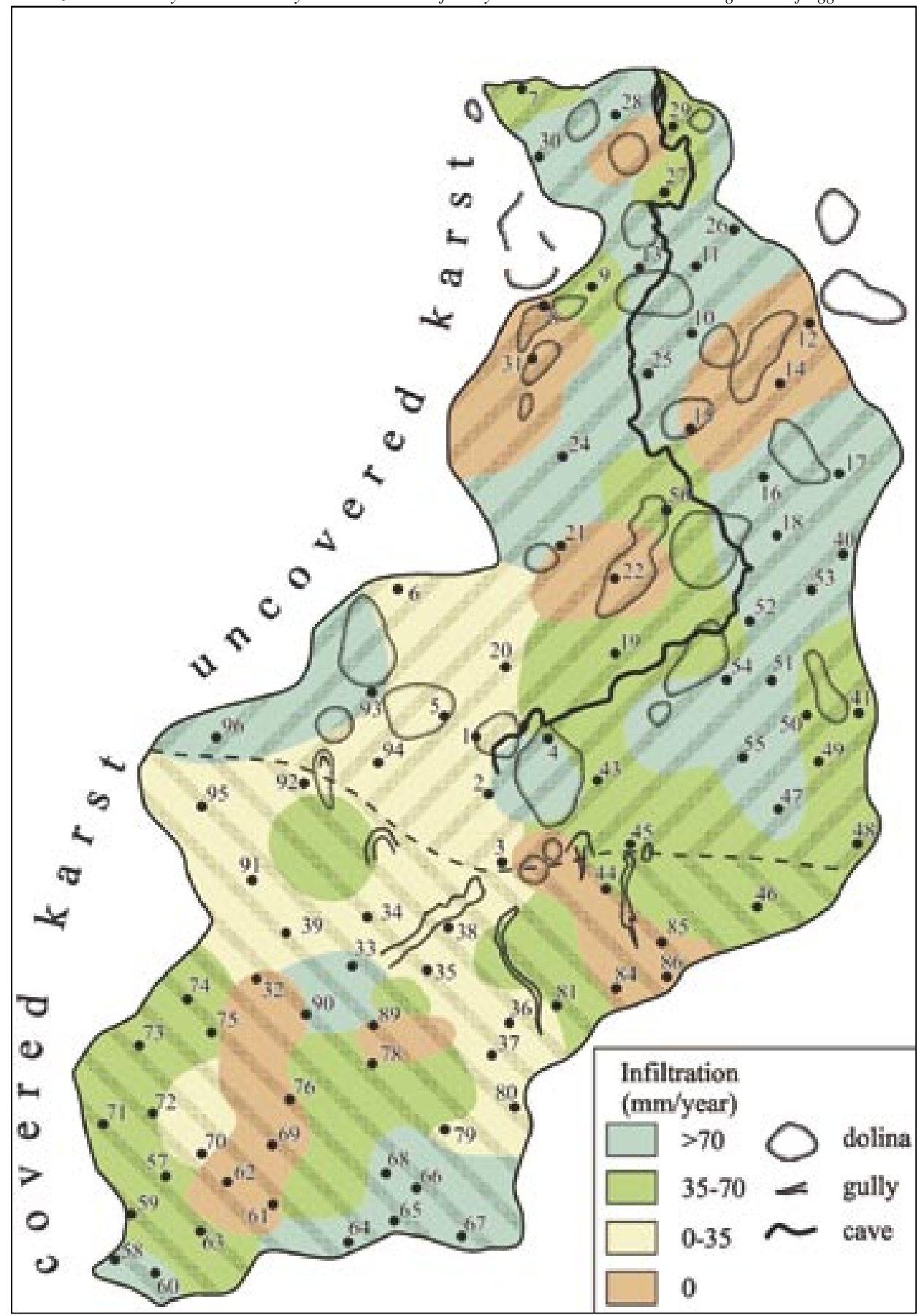

II. Map Catchment basin of Béke cave with the sample points. 


\begin{tabular}{|c|c|c|c|c|c|c|c|c|c|c|c|c|c|c|c|}
\hline$\stackrel{\circ}{a}$ & $\infty$ & 蛋 & $\stackrel{\infty}{0}$ & $\stackrel{\infty}{\circ}$ & 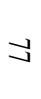 & $\vec{\omega}$ & $\curvearrowright$ & $\breve{\infty}^{\prime \prime}$ & us & uू & $u^{\prime \prime}$ & $\stackrel{L}{\perp}$ & 岕 & \multicolumn{2}{|c|}{ 总愛 } \\
\hline$\stackrel{\varpi}{\frac{\sigma}{d}}$ & 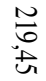 & 岕 & $\begin{array}{l}\bar{\omega} \\
\stackrel{D}{\infty}\end{array}$ & $\begin{array}{l}\vec{\infty} \\
\stackrel{w}{\omega} \\
\stackrel{\omega}{\omega}\end{array}$ & $\frac{\bar{N}}{80}$ & 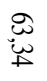 & 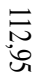 & $\stackrel{\vec{v}}{\vec{y}}$ & $\begin{array}{l}\text { Uू } \\
\text { ğ }\end{array}$ & $\begin{array}{l}\text { 高 } \\
\text { 剀 }\end{array}$ & 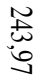 & $\begin{array}{l}\text { 岕 } \\
\text { ö }\end{array}$ & $\begin{array}{l}\omega \\
\omega \\
\omega \\
\omega\end{array}$ & 픽 & ふ \\
\hline $\begin{array}{l}\text { : } \\
\text { 告 }\end{array}$ & 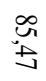 & $\underset{\vec{u}}{\vec{u}}$ & $\begin{array}{l}\stackrel{\omega}{ \pm} \\
\stackrel{\sigma}{\sigma}\end{array}$ & $\begin{array}{l}\text { 怘 } \\
\stackrel{\circ}{\circ}\end{array}$ & $\underset{\sim}{\omega}$ & $\begin{array}{l}\text { N్心 } \\
\text { 足 }\end{array}$ & 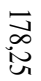 & 茜 & $\underset{\text { స్ }}{\Xi}$ & $\begin{array}{l}w^{\prime} \\
\ddot{\alpha}\end{array}$ & $\stackrel{\omega}{\omega}$ & $\stackrel{\widetilde{\Omega}}{\varrho}$ & $\begin{array}{l}\infty \\
\infty\end{array}$ & § & 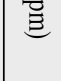 \\
\hline$\stackrel{\omega}{\omega}$ & $\underset{\mathrm{N}}{\vec{J}}$ & 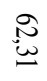 & 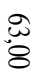 & $\frac{9}{8}$ & $\stackrel{\infty}{\stackrel{+}{8}}$ & $\begin{array}{l} \pm \\
\pm\end{array}$ & $\begin{array}{l}\text { U్ర } \\
\text { "ర }\end{array}$ & $\begin{array}{l}\vec{w} \\
\text { is }\end{array}$ & $\begin{array}{l}\text { న } \\
\infty \\
\infty \\
\infty\end{array}$ & $\begin{array}{l}\stackrel{t}{0} \\
\text { 㞻 }\end{array}$ & 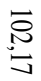 & 胥 & مِّ & 픽 & 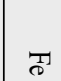 \\
\hline \&̊ & 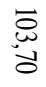 & 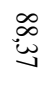 & 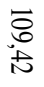 & 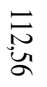 & $\underset{\infty}{\stackrel{\sigma}{\sigma}}$ & 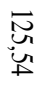 & $\underset{\omega}{\stackrel{\omega}{\omega}}$ & $\begin{array}{l}\Xi \\
\stackrel{\circ}{8}\end{array}$ & $\underset{\sim}{\stackrel{8}{*}}$ & $\begin{array}{l}\ddot{8} \\
\dot{8} \\
\ddot{8}\end{array}$ & $\stackrel{8}{8}$ & 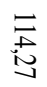 & 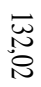 & @ & \\
\hline $\begin{array}{l}\text { प्र } \\
\text { U్ర }\end{array}$ & $\begin{array}{l}+t \\
\stackrel{5}{\infty}\end{array}$ & 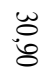 & 岕 & 总 & 芯 & 岕 & 怘 & $\underset{\omega}{\tilde{\sigma}}$ & $\underset{\stackrel{F}{+}}{\stackrel{L}{+}}$ & $\underset{+}{\stackrel{N}{\infty}}$ & $\begin{array}{l}\mathscr{C}_{0} \\
\stackrel{\infty}{0}\end{array}$ & $\underset{\infty}{\infty}$ & $\begin{array}{l}\text { w } \\
\text { to }\end{array}$ & $\underset{3}{9}$ & 3 \\
\hline $\begin{array}{l}\text { Uి } \\
0 \\
0 \\
\infty \\
2\end{array}$ & 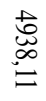 & 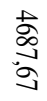 & 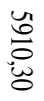 & $\begin{array}{l}\frac{+}{+} \\
\frac{1}{\sigma} \\
a\end{array}$ & $\begin{array}{l}\overrightarrow{0} \\
8 \\
\text { 崩 }\end{array}$ & 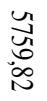 & $\begin{array}{l}\stackrel{\leftrightarrow}{\vec{W}} \\
\stackrel{\infty}{\infty} \\
\stackrel{\infty}{\infty}\end{array}$ & 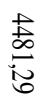 & $\begin{array}{l}u^{\prime} \\
\infty \\
w_{u}^{\prime}\end{array}$ & $\begin{array}{l}\underset{\sim}{\tilde{U}} \\
\stackrel{\vec{H}}{\vec{t}}\end{array}$ & $\begin{array}{l}\text { 志 } \\
\infty \\
\infty \\
\dot{\Phi}\end{array}$ & 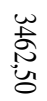 & 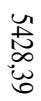 & 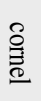 & \\
\hline $\begin{array}{l}\text { 苟 } \\
\stackrel{0}{+}\end{array}$ & 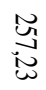 & 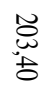 & $\underset{+}{\stackrel{N}{ \pm}}$ & $\begin{array}{l}\text { Un } \\
\stackrel{1}{N} \\
\infty\end{array}$ & $\begin{array}{l}+ \\
\stackrel{\infty}{\circ} \\
\stackrel{0}{0}\end{array}$ & 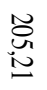 & $\begin{array}{l}\overrightarrow{+} \\
\infty \\
\stackrel{\infty}{\infty}\end{array}$ & 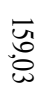 & $\frac{\sqrt[n]{t}}{\ddot{f}}$ & 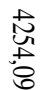 & $\begin{array}{l}\frac{N}{0} \\
\text { है }\end{array}$ & $\begin{array}{l}\text { ్ㅗㅇ } \\
\text { 心 } \\
\text { N }\end{array}$ & $\begin{array}{l}\vec{\circ} \\
\stackrel{\infty}{+\infty} \\
\infty\end{array}$ & 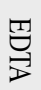 & 8 \\
\hline 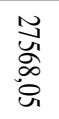 & $\begin{array}{l}\text { 心్心 } \\
\text { 心్ } \\
\text { స్ }\end{array}$ & 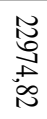 & $\begin{array}{l}\text { 岕 } \\
\text { U్ర } \\
\stackrel{\infty}{\infty}\end{array}$ & 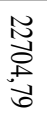 & $\begin{array}{l}\text { 壱 } \\
\text { ? } \\
\text { 足 }\end{array}$ & 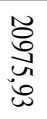 & 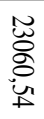 & 芯 & 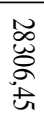 & 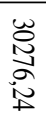 & 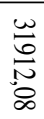 & 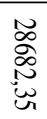 & 岕 & 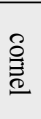 & 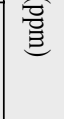 \\
\hline $\begin{array}{l}\vec{A} \\
\text { 䓌 }\end{array}$ & $\begin{array}{l}\breve{w} \\
\stackrel{+}{\sigma} \\
\sigma\end{array}$ & $\underset{\tilde{\sigma}}{\tilde{\sigma}}$ & $\stackrel{\vec{a}}{\exists}$ & $\underset{\omega}{\vec{w}}$ & 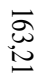 & $\begin{array}{l}\overrightarrow{0} \\
\text { Nㅓㄴ }\end{array}$ & 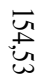 & 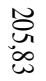 & 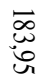 & $\begin{array}{l}\text { 岕 } \\
\text { 志 }\end{array}$ & $\stackrel{\overline{\vec{N}}}{\mathrm{~N}}$ & 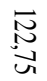 & $\begin{array}{l}\overline{\text {. }} \\
\text { 空 }\end{array}$ & 픽 & $=$ \\
\hline$\underbrace{\prime}_{\vec{D}}$ & $\begin{array}{l}\mathbb{N} \\
\text { N }\end{array}$ & 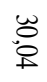 & "ü & 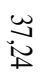 & $\begin{array}{l}\text { in } \\
\text { o }\end{array}$ & $\underset{\text { 志 }}{t}$ & $\begin{array}{l}\text { पू } \\
\text { 崩 }\end{array}$ & $\stackrel{\infty}{=}$ & ن. & $\begin{array}{l}w \\
\infty \\
\perp\end{array}$ & $\stackrel{+}{8}$ & 足 & $\infty$ & §. & \\
\hline 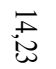 & $\frac{7}{8}$ & $\begin{array}{l}\text { : } \\
\text { ĩ }\end{array}$ & w్w & $\underset{\dot{c}}{\stackrel{\square}{0}}$ & \begin{tabular}{l}
3 \\
$\stackrel{3}{3}$ \\
\hdashline
\end{tabular} & $\stackrel{\bar{a}}{\stackrel{\partial}{\partial}}$ & 芯 & $\begin{array}{l}10 \\
8\end{array}$ & $\underset{\infty}{+\infty}$ & 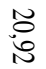 & $\underset{+\infty}{w}$ & $\stackrel{N}{ \pm}$ & $\frac{2}{8}$ & 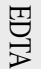 & 지 \\
\hline 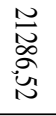 & 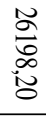 & 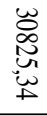 & 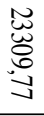 & 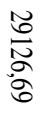 & 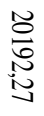 & 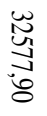 & $\stackrel{\Xi}{\Xi}$ & 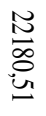 & 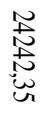 & $\begin{array}{l}\underset{ٍ}{0} \\
\stackrel{\infty}{0} \\
0\end{array}$ & \begin{tabular}{l}
$\tilde{+}$ \\
\multirow{2}{*}{} \\
$\infty$ \\
$\infty$ \\
0 \\
0
\end{tabular} & 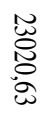 & 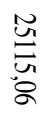 & 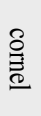 & \\
\hline 古 & 总 & $\vec{u}$ & yू & $\stackrel{\sim}{\sim}$ & 岕 & 兄 & 忩 & $\vec{E}$ & $\Xi$ & $\stackrel{\omega}{ \pm}$ & 0 & $\stackrel{\omega}{\sim}$ & $\ddot{\infty}$ & $\underset{7}{3}$ & $\S$ \\
\hline$\vec{\omega}$ & 悹 & $\stackrel{u}{0}$ & 䜣 & $\stackrel{\circ}{\circ}$ & $\ddot{~}$ & 空 & 岌 & 吢 & \pm & $\ddot{\circ}$ & $\stackrel{\overrightarrow{0}}{a}$ & $\stackrel{\overrightarrow{0}}{\circ}$ & $\ddot{\infty}$ & @) & \\
\hline $\bar{\sigma}$ & 芯 & $\vec{\infty}$ & $\sigma$ & $\stackrel{N}{\infty}$ & $\stackrel{\omega}{\infty}$ & $\ddot{8}$ & 芯 & 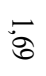 & $\stackrel{\oplus}{\omega}$ & $\stackrel{n}{8}$ & is & ש్ర & $\stackrel{\sim}{\perp}$ & 夏 & $\cong$ \\
\hline $\overrightarrow{\tilde{8}}$ & $\underset{\mathscr{D}}{\tilde{+}}$ & $\begin{array}{l}\infty \\
\text { i }\end{array}$ & 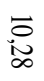 & $\underset{\sim}{\stackrel{\omega}{\omega}}$ & $\overrightarrow{\breve{b}}$ & 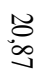 & $\stackrel{\mathbb{U}}{\stackrel{N}{\sigma}}$ & 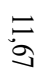 & $\stackrel{\Xi}{\Xi}$ & बें & 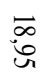 & $\stackrel{\vec{\not}}{\vec{\perp}}$ & $\overrightarrow{⿱ 亠}$ & $\stackrel{\varrho}{\Xi}$ & 5 \\
\hline
\end{tabular}


R. Kaszala \& I. Bárány-Kevei \& K. Polyák: Further dates of heavy metal content on the soil and vegetation of Aggtelek Karst

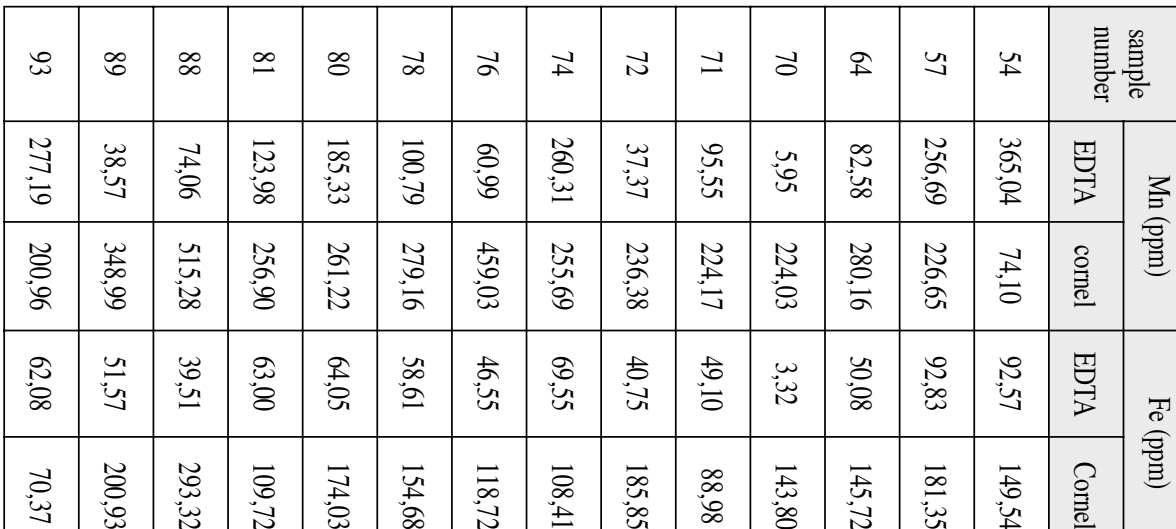

$\frac{a}{5}$

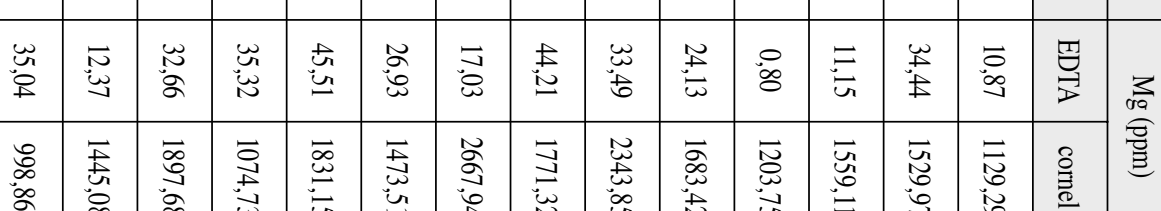

站

\begin{tabular}{|c|c|c|c|c|c|c|c|c|c|c|c|c|c|c|}
\hline 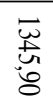 & $\begin{array}{l}\text { 我 } \\
\ddot{d}\end{array}$ & $\begin{array}{l}\stackrel{0}{心} \\
\stackrel{\infty}{0}\end{array}$ & $\underset{+}{\stackrel{\tilde{t}}{\neq}}$ & 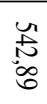 & $\begin{array}{l}0 \\
\stackrel{0}{\circ}\end{array}$ & 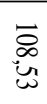 & 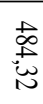 & $\stackrel{\omega}{\sim}$ & $\begin{array}{l}\stackrel{\sim}{\sim} \\
\stackrel{\perp}{\perp}\end{array}$ & $\vec{A}$ & $\begin{array}{l}\vec{W} \\
\stackrel{ \pm}{ \pm}\end{array}$ & 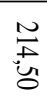 & $\begin{array}{l}\text { N్ర్ } \\
\text { 心్త } \\
\text { N }\end{array}$ & 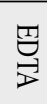 \\
\hline$\underset{w}{\omega}$ & 莺 & $\stackrel{t}{0}$ & 吕 & ü & 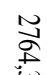 & హ్ర్ర్ & & 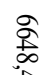 & $\underset{⿱ 士 心}{ \pm}$ & $\underset{.]}{w}$ & 鸢 & 岕 & ب్. & § \\
\hline 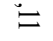 & 必 & $\not \circ$ & $\bar{\square}$ & $\infty$ & w & 古 & 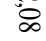 & \pm & i & $\stackrel{J}{J}$ & $\stackrel{\sim}{+}$ & $\not$ & 识 & D. \\
\hline
\end{tabular}

i

胥

\begin{tabular}{|c|c|c|c|c|c|c|c|c|c|c|c|c|c|c|}
\hline$\stackrel{\simeq}{\simeq}$ & $\ddot{u}$ & $\doteq$ & $\stackrel{\sim}{\triangleleft}$ & $\stackrel{J}{J}$ & సু & to & $\stackrel{\sim}{\perp}$ & 岕 & బै & "ু' & 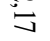 & li & $\vec{u}$ & $\overrightarrow{8}$ \\
\hline & $\mp$ & $\underset{\not}{\mathbb{A}}$ & ü & 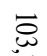 & $\infty$ & שై & $\sigma$ & 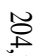 & $\vec{r}$ & 2 &. & $\bar{\omega}$ & $\vec{\omega}$ & $\S$ \\
\hline
\end{tabular}

(2)

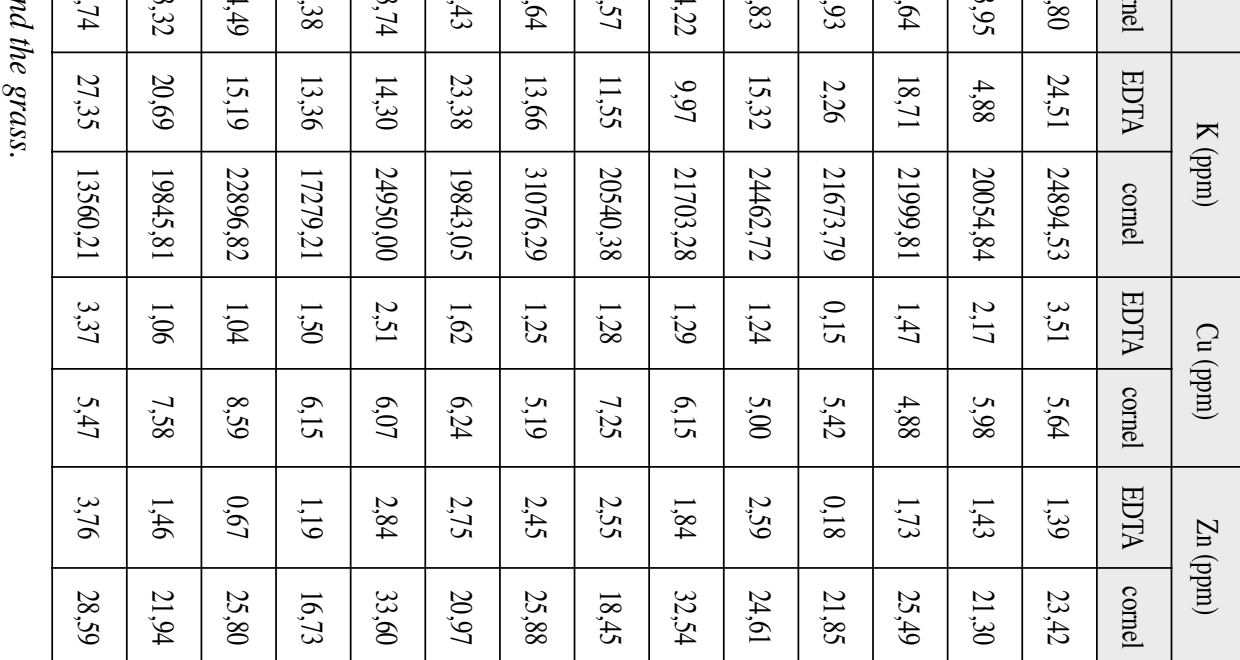


ஜ

禹

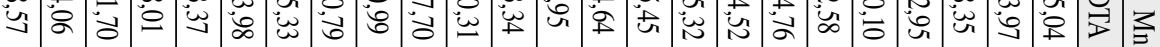

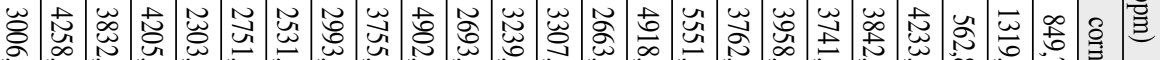
品

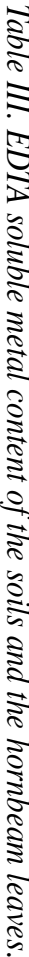

u

$\frac{8}{5}$

"ె口 山े

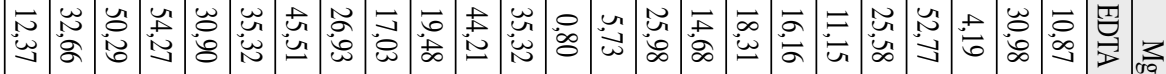

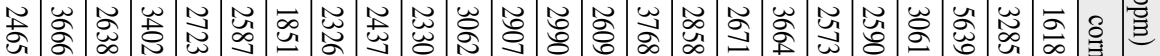

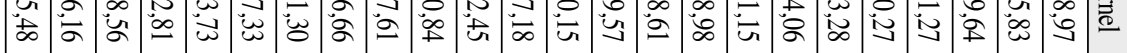

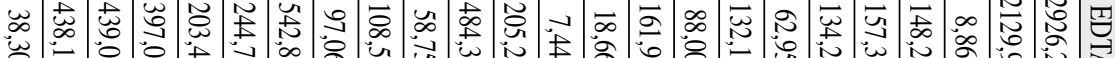
岩

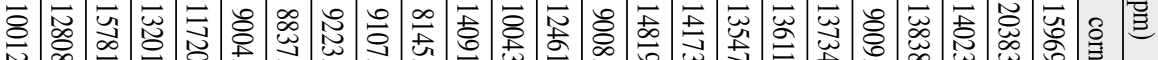

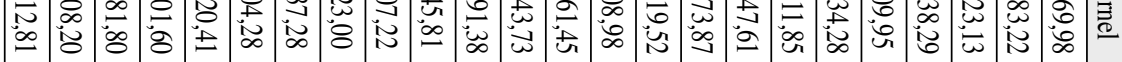

坣

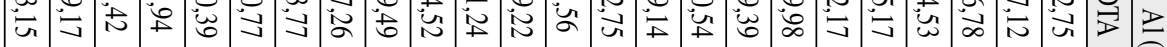
岕

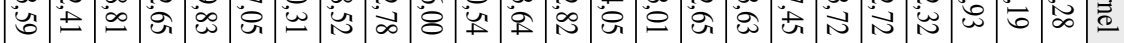

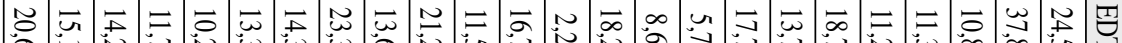

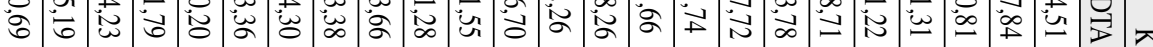

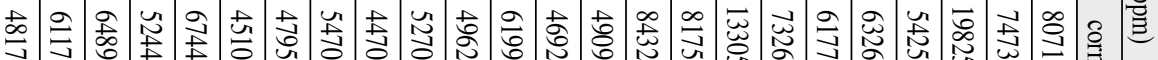

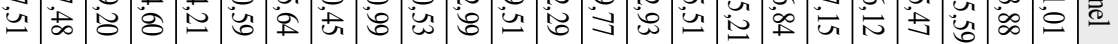

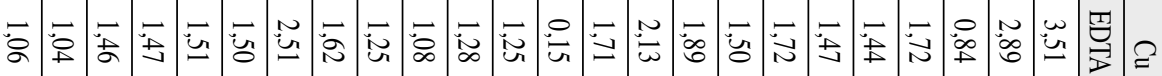

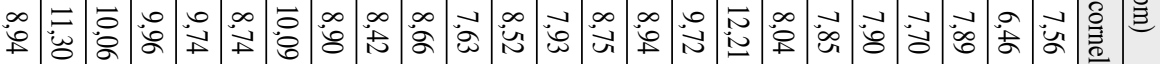
去

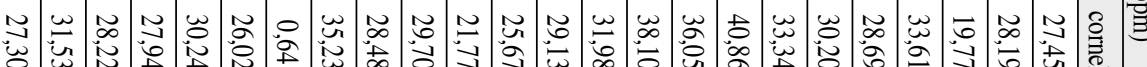




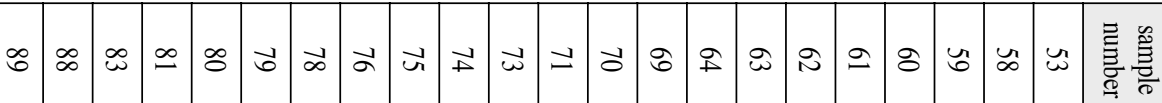

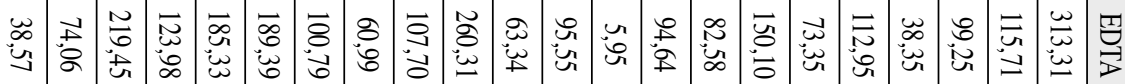

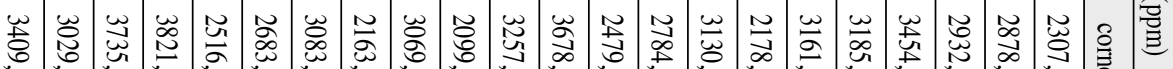

定

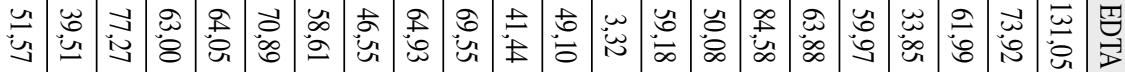

$\rightarrow$ 의

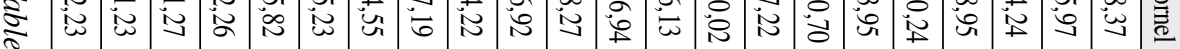

$\gtrless$

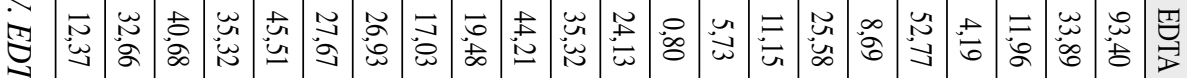

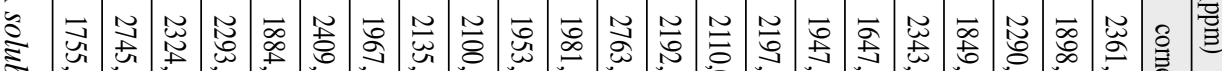

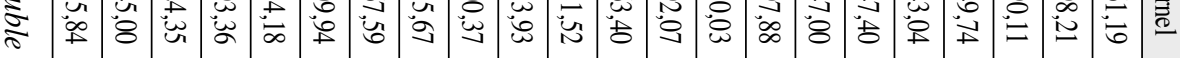

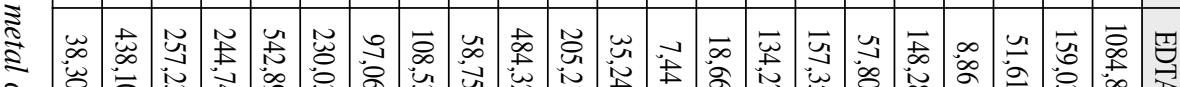

3 岩

ङ

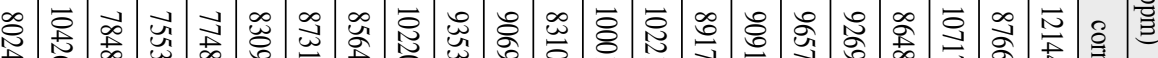

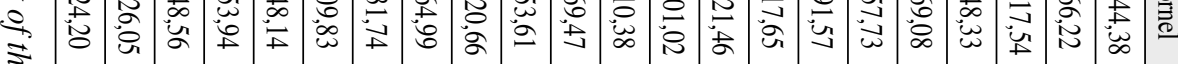

क

岕

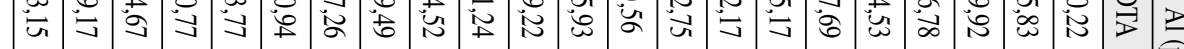

$\S$

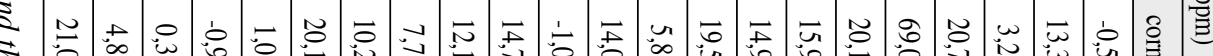

亲

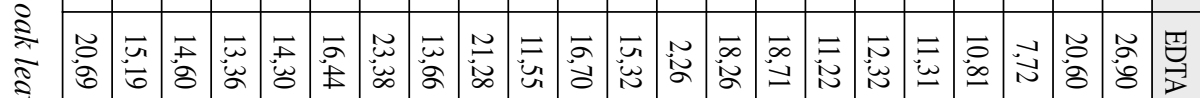

कृ

\begin{tabular}{|c|c|c|c|c|c|c|c|c|c|c|c|c|c|c|c|c|c|c|c|c|c|c|c|}
\hline & $\begin{array}{l}\text { ⿹े山े } \\
\infty \\
\text { 屯 }\end{array}$ & $\begin{array}{l}\stackrel{H}{\exists} \\
\partial \\
\infty \\
\infty\end{array}$ & 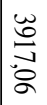 & 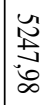 & $\begin{array}{l}\text { w } \\
\vec{\sigma} \\
\stackrel{\sigma}{\sigma}\end{array}$ & 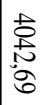 & 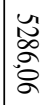 & 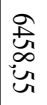 & 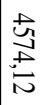 & 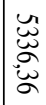 & 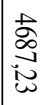 & 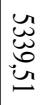 & $\begin{array}{l}0 \\
0 \\
\stackrel{0}{ \pm} \\
0 \\
0\end{array}$ & 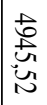 & 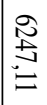 & 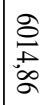 & $\mid \begin{array}{l}u \\
\vec{u} \\
\text { 岕 } \\
\end{array}$ & 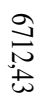 & 岂 & 岂 & & @ & \\
\hline & 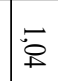 & N & $\ddot{b}$ & $\stackrel{\sim}{\sim}$ & $\tilde{w}$ & 兽 & 兌 & $\infty$ & 沛 & 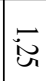 & 芒 & $\stackrel{0}{\dot{v}}$ & 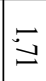 & \pm & $\overrightarrow{+}$ & 5 & $\stackrel{\sim}{N}$ & $\stackrel{\circ}{\infty}$ & 芯 & $\overrightarrow{ \pm}$ & $\stackrel{w}{\infty}$ & 四 & \\
\hline & $\stackrel{\bar{N}}{\mathbb{E}}$ & 吕 & $\underset{\infty}{\infty}$ & $\underset{\infty}{\stackrel{\infty}{\circ}}$ & $\bar{\omega}$ & $\begin{array}{ll}0 \\
\ddot{4}\end{array}$ & 电 & $\stackrel{\Xi}{\stackrel{0}{0}}$ & $\begin{array}{l}\bar{i} \\
\text { ind }\end{array}$ & ळ & $\begin{array}{l}\overrightarrow{0} \\
\text { 呆 }\end{array}$ & 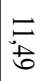 & 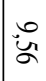 & $\begin{array}{l}\sigma \\
0 \\
0\end{array}$ & 㳘 & $\because 0$ & 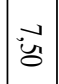 & $\ddot{0}$ & 心 & $\stackrel{a}{\star}$ & 鸰 & 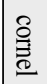 & \\
\hline & $\stackrel{\circ}{9}$ & 空 & 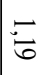 & 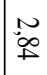 & 崖 & $\stackrel{N}{\sim}$ & $\begin{array}{l}n \\
\stackrel{n}{*}\end{array}$ & 盛 & 畓 & تृ & 빙 & $\stackrel{0}{\infty}$ & $\stackrel{\sim}{\tilde{u}}$ & $\stackrel{\bar{\omega}}{\omega}$ & $\ddot{\partial}$ & $\breve{\infty}_{\infty}$ & 芯 & $\check{N}$ & 芯 & 5 & $\underset{\omega}{\omega}$ & 雨 & \\
\hline & $\underset{\substack{N \\
\infty}}{\bar{\infty}}$ & $\overrightarrow{2}$ & $\infty$ & $\stackrel{\sim}{\circ}$ & $\stackrel{0}{0}$ & $\vec{\Delta}$ & $\begin{array}{l}0 \\
0 \\
0 \\
0\end{array}$ & 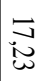 & $\underset{0}{\sigma}$ & $\overrightarrow{\ddot{n}}$ & 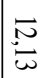 & $\begin{array}{l}N \\
N \\
\infty \\
\infty\end{array}$ & $\stackrel{\Xi}{\ominus}$ & $\begin{array}{l}\infty \\
\ddot{0} \\
+\end{array}$ & $\begin{array}{l}\vec{\nabla} \\
\frac{\vec{\theta}}{0}\end{array}$ & $\underset{f}{F}$ & 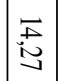 & $\begin{array}{l}\vec{\infty} \\
\dot{l} \\
\text { in }\end{array}$ & बूa & $\frac{N}{N}$ & $\begin{array}{l}\omega \\
\omega \\
\omega\end{array}$ & 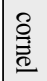 & \\
\hline
\end{tabular}




\section{DISCUSSION}

The examination of the heavy metal content of the greenery was started much more lately than the investigations of the heavy metal contents of the soils. This is the reason, that there are not so many literatures about the metal content of the greenery.

In plants the most common route of exposure is through the roots. Ions and organic molecules contact roots via the transpiration steam, diffusive transport and microbially facilitated transport. Once at the root surface, soluble contaminants have the potential to enter into root tissue through the transpiration steam or through a range of mechanisms that era designed to facilitate nutrient uptake EHLERS L. J. (2003). The phytophysiologyst distinguish plants by their uptake strategy. By right of this we can find species which are accumulate, exclude or indicate (BAKER, 1981).

At 1970 Tölgyesi and his contributors were find that on the same conditions the one year-old terophyton and hemikriptophyton plants generally contain more trace elements than the perpetual ones. They wrote that the species of Asteraceae accumulate mainly the copper, the species of Caryophyllaceae mainly the manganese and the species of Salicaceae mainly zinc TÖLGYESI et al. (1970). In case of wood-bodies was investigate the amount of transported trace element to the leaves SZALAI (1998). PETERSON $(1971,1975)$ was investigated the ratio of plant/soil at 12 species amount of $\mathrm{Zn}, \mathrm{Pb}, \mathrm{Cd}$ and $\mathrm{Cu}$.

The greenery of karstic area in Hungary was examined BÁRÁNY-MEZŐSI (1999) at first. Based on the determination of the authors the heavy metal content of the greenery converges to the heavy metal content maximum of the soils, and at lower soil $\mathrm{pH}$ the greenery has higher metal content.

According to FÜLEKY (1999) the examined plants contain metals in the following concentrations: Mn: 20-500 ppm, Fe: 50-250 ppm, Mg: 1000-5000 ppm, Ca: 1000-40000 ppm, Al: 200 - 4000 ppm, K: 10000-50000 ppm, Cu: 2-20ppm, ppm, Zn: 25-150ppm.

KÁDÁR I. (1995) measured the following concentrations in the leaves of oak tree: Mn: 41 ppm, Fe: 487 ppm, Al: 283 ppm, Cu: 9 ppm Zn: 44 ppm.

On the I. Table we can find the measure result of the EDTA soluble metal content of the soils and the cornel leaves metal content. The leaves manganese, iron, magnesium, calcium, potassium and copper content are in accordance with the literature date, but the aluminium and the zinc contents are lower than we expect.

On the II. Table we can see the measure result of the EDTA soluble metal content of the soils and the grass metal content. These date have accordance with the literature date except for the aluminium which has lower values.

On the III. Table we can find the measure result of the EDTA soluble metal content of the soils and the hornbeam leaves metal content. The manganese content of the leaves is higher than the literature values, the other elements content agree with them.

On the IV. Table we can find the measure result of the EDTA soluble metal content of the soils and the oak leaves metal content. In case of the oak leaves metal content we can see, that the content of manganese is much more higher, than the literature value. The aluminium, potassium, copper and zinc have lower values, and the iron, magnezium and calcium content is according to the relevant literature. 


\section{CONCLUSION}

On the basis of analyse of EDTA soluble metals in soil and plants, we concluded:

- Manganese: In case of cornel the EDTA soluble Mn content of the soil is higher than the Mn content of the cornel leaves. The other plants we can see that the leaves Mn content is higher than in the soil.

- Iron: In case of oak at seven samples the soils iron content is higher than the iron content of the plants. The other cases and the other plants the soil content is lower than the plant concentration.

- Aluminium: In the soil EDTA soluble Al content is higher than the Al concentration of grass, oak and cornel, but in nineteenth samples of hornbeam the $\mathrm{Al}$ concentration is higher in the plant and only five samples have higher $\mathrm{Al}$ concentration in the soils.

- Magnesium, potassium, calcium: As these elements are macro elements for the plants, for this case we can see in every species higher concentrate in the plants than in the EDTA soluble part of soils

- Cupper, zinc: In every samples have higher plant concentrate. It is also normal as these elements are microelements for the plants in every species.

\section{LITERATURE}

BAKER, A. J. M. (1981): Accumulators and Excluders-Strategies in the Response of Plants to Heavy Metals. Journal of Plant Nutrition, 3(1-4). p. 643-654.

BÁRÁNY-KEVEI, I.- MEZŐSI, G.(1999).: The relationships between soil chemistry and the heavy metal content of vegetation on karsts. In.: Essays in the ecology and conservation of karst. (Ed. Bárány-Kevei, I., Gunn, J.) p. 47-53.

EHLERS, L.J.(2003): Bioavailability of contaminants in soils and sediments. National Research Council, National Academies Press, Washington p. 180-182.

FÜLEKY GY. (1999): Tápanyag-gazdálkodás (Alimentary substances husbandry) - Mezőgazda Kiadó p. 70-80

KÁDÁR I. (1995): Környezet- és Természetvédelmi Kutatások. Research of Conservation and Environmental Protection) - MTA Agrokémiai és Talajtani Kutató Intézet, Budapest, p. $131-132$

LAKANEN, E. - ERVIÖ, R. (1971) A comparison of eight extract ant for the determination of plant available micronutrients in soil. Act Agra. Fen. 123. p 223-232.

PETERSON, P. J. (1971): Unusual accumulations of elements by plants and animals. Sci.Prog., Oxf.59, p. 505-526.

PETERSON, P. J. (1975): Element accumulation by plants and their tolerance of toxic mineral soil. Proc.Int. Conf. On Heavy metals in the Environment. Valhi, Toronto, Canada p. 39-54.

ROWELL, D.L. (1994): Soil Science: Methods and Applications. Longmann Group UK Limited, p. 350 .

SZALAI Z. (1998): Nehézfémek teljes ülepedésének meghatározási lehetőségei talaj- és növényminták analízisének segítségével. Földrajzi Értesító, 48. p. 515-523.

TÖLGYESI GY., KÁRPÁTI I., KÁRPÁTI I-NÉ (1970): Savanyú és meszes homokpuszták növényzetének makro- és mikrotápanyagfelvétele. Agrokémia és talajtan, 19. p. 55-65. 
